fragments are transferred to the egg and parthenogenetic development of the egg occurs. The chromosome number of the egg or early embryo then doubles and finally some of the fragments are incorporated into, or associated with, the maternal genome. A crucial point to be investigated is whether the chromosome doubling and parthenogenetic development that occur so readily in these Nicotiana species, can occur as readily in other genera.

The size and nature of the chromosome fragments from the pollen parent, and the mechanism of their integration or association is of great interest. Genetical studies of linked markers and of segregation patterns in later generations will help to determine the size and location of the associated or integrated fragments, and physical characterization of particular
DNA fragments will be feasible. A general assumption of radiation cytogenetics is that broken ends of chromosomes rejoin only with other broken ends; since the maternal parent is unirradiated such ends will not be available for rejoining with the fragments produced in the pollen. How else might integration occur, if this is indeed what happens? Fragments could be incorporated during replication, or during the exchange events that ultimately become recognized as sister chromatid exchanges; if this is so, then the average size of the fragments would be expected to be small. Transposable elements of the kind first recognized in McClintock's work on maize, and physically characterized, for example, in Copia in Drosophila, must have a chromosomal organization which allows integration into, and excision from, eukaryotic chromosomes; but it seems unlikely that random fragments induced by radiation would have a similar organization. One of many puzzles posed by the Nicotiana results is the frequency with which transfer of attributes occurs; 6 out of $97 \mathrm{M}_{2}$ progeny generated after a 20 $\mathrm{krad}$ dose of $\gamma$ radiation to the pollen closely resembled the maternal parent yet had the black ovary character from the paternal parent. It remains to be seen whether this is stable in later generations and whether black ovary is typical in its rate of transfer; if it is, then this represents a high rate of integration or association. Clearly there are a large number of issues to be resolved but these investigations by Pandey and by Jinks and his colleagues should now provide an incentive for a great number of workers to re-examine the basis and practical application of the phenomenon of gene transfer.

\title{
Cell sorting, cell differentiation and cyclic AMP
}

from Adrian Tsang

IN the slime mold Dictyostelium discoideum, cyclic AMP is now believed to be directly involved in the processes of cell sorting and differentiation, as well as acting as a chemotactic agent in mediating aggregation. This is one of the conclusions that emerged from a recent meeting* on the development of cellular slime molds.

Amoebae of $D$. discoideum multiply as isolated cells. The developmental programme is set in motion at the onset of starvation, and after about six hours the amoebae aggregate in response to cyclic AMP signals. The multicellular structures, called slugs, which are formed after aggregation are differentiated along the anterior-posterior axis. The anterior, prestalk region gives rise to the stalk cells of the terminally differentiated fruiting body, while the cells of the posterior region are the precursors of the mature spores. This formation of a patterned arrangement of new cell types has obvious analogues in the development of higher eukaryotes.

It has been known for some years that prestalk and prespore cells can sort out, but it is not entirely clear whether sorting out precedes differentiation or is a consequence of it (Forman and Garrod $J$. Embryol. exp. Morph. 40; 229, 1977). The answer to this question is important because it will indicate whether or not the position a cell occupies in the aggregate has an effect on its differentiation. Making use of the observation that amoebae grown in a non-glucose medium, when mixed with amoebae grown in a glucose-containing medium, differentiate preferentially into

*The US-Japanese conference on the development of cellular lime molds held in San Diego. California in February was organized by 1. Takcuchi (Kyoto University) and D. Francis (Universily of Delaware). prestalk cells (Leach et al. J. Embryol. exp. Morph. 29; 647, 1973), Takeuchi (Kyoto University) examined the relationship between sorting out and differentiation. $\mathrm{He}$ mixed ${ }^{3} \mathrm{H}$-labelled, non-glucose-grown amoebae with glucose-grown amoebae and allowed them to form agglomerates in submerged conditions. At various times the agglomerates were sectioned and stained with anti-spore serum to locate the prespore cells, and the same sections were autoradiographed to locate non-glucosegrown cells. Takeuchi found that the prespore cells began to differentiate long before the labelled and unlabelled cells were sorted out, suggesting that cell sorting is not a prerequisite for differentiation. This thesis is supported by a separate experiment performed by Okamoto (Kyoto University) in which dissociated aggregative cells were shaken at high speed. Under these conditions, small clumps of cells were formed and the prespore cells in the clumps were found to be randomly distributed. As no cell sorting can occur in these small clumps of cells, the above data indicate that $D$. discoideum cells can differentiate in the absence of cell sorting. In other words, whether a cell differentiates into a prestalk or a prespore cell may not depend on the position it occupies in the multicellular aggregate.

Cellular sorting out has often been attributed to differential adhesion. That this may not be the whole story in $D$. discoideum was demonstrated by Sternfeld (Albert Einstein College) and Takeuchi. Sternfeld found that when dissociated slug cells were allowed to form clumps in suspension, a new pattern was formed with the prestalk cells in the centre of the clump. However, if the culture medium contained cyclic AMP, the prestalk cells were found on the periphery. Moreover, Takeuchi showed that prestalk cells possess higher motive force than do prespore cells (Inouye and Takeuchi J. Cell Sci. 41; 53, 1980). Although these experiments do not rule out the possible participation of differential adhesion in sorting out, they do strongly support the earlier suggestion of Matsukama and Durston (J. Embryol. exp. Morph. 50; 243, 1979) that cell sorting is guided by differential chemotaxis to cyclic AMP.

Evidence accumulated over the past decade indicates that cyclic AMP participates in the differentiation of stalk and spore cells. Both Sussman (University of Pittsburgh) and Rutherford (Virginia Polytechnic Institute) showed that the potential of establishing a cyclic AMP gradient exists in the multicellular aggregate. Blumberg (MIT) and Okamoto provided additional evidence that the accumulation and the maintenance of postaggregative gene products require cyclic AMP (Town and Gross Devl Biol. 63; 412, 1978), and there is direct evidence that cyclic AMP affects the expression of developmental gene products by altering the rates of transcription of specific genes (Williams et al. Proc. natn. Acad. Sci. U.S.A. 77; 7171, 1980). What remains obscure is the role of cyclic AMP in cell differentiation. Is it a general effector of gene expression, a morphogen which channels the cells into specific differentiative pathways, a maturation factor for stalk and spore cells or a combination of these?

Adrian Tsang is at the Imperial Cancer Research Fund Mill Hill Laboratories, London, UK. 